\title{
Restitution Movement for a Low Cost Driving Simulator
}

\author{
L. Nehaoua, H. Arioui, H. Mohellebi and S. Espie
}

\begin{abstract}
This paper deals with motion control problem for a 2 DOF small driving simulator. The main idea is to test and compare performances of different Washout algorithms applied to such platform category. The experimentations allow us to have the best compromise between quality of the human perception, implementation complexity and platform architecture type.

Implementation of different Washout algorithms (optimal, adaptive and classical one) are discussed. For an objective comparison, the classic algorithm was synthetized so as to take into account the human perception thresholds and the platform workspace limitations. A modelling of a second DOF (seat tilting) is given and simulated to show the inertial effect of the seat movement. Finally, phsycophysics study, experimental results and conclusion are given.
\end{abstract}

\section{INTRODUCTION}

The increase of road accident and the governomental institution to road safety urged the researchers in the field of vehicle, in particular driving simulation, to be closely interseted. After multiple investigations, it turns out that the driving training can reduce considerably the human and materials damages. Therefore, the solution of the use of driving simulators techniques was held.

More tecnically, the use of virtual reality techniques in the domain of driving simulation is not for nowadays. Flight simulators had the first which explore this, for constructing motion base simulator to provide the pilot with the most relevant inertial cues for more realistic simulation environment. After, numerous driving vehicle simulators have been developed with different purposes by many research laboratories, and industrial companies, most often for human behavior study, or for testing new models and prototypes.

It is true that driving a vehicle is a visual task. In fixed-base driving simulators, the driver manipulates a set of driving controls such accelerating, braking, steering to receive visual cues corresponding to the actual driving situation. Although, providing the driver with inertial cues (movement) would improve dramatically the simulation realism. Consequently, for a simulation to be realistic, a platform with motion base which may exert a linear acceleration and angular velocities on the simulator's cabin is required, in addition, to the use of commonly visual environment, audio and other haptic feedbacks [1].

Vehicle acceleration can not be reproduced totally, a compromise must be realized between the restitution of inertial indices and the maintain of the platform in its workspace

L. Nehaoua and H. Arioui: IBISC CNRS Fre 2873, 40 rue Pelvoux 91020, Evry, France. email: nehaoua, arioui@iup.univ-evry.fr

H. Mohellebi and S. Espie: INRETS, 2 Av Gl Malleret-Joinville, 94114, Arceuil, France. email: mohellebi, espie@inrets.fr limits. Thus, many command strategies were developed first for flight simulators area [2], [3], [4], [5], [6], [7]. Since that problematic is similar to the driving simulator, the application of these algorithms is direct [8]. However, some characteristics of driving must be taken into consideration. The dynamics of a vehicle are indeed different from those of an airplane, and the 6 DOF acceleration variations in a vehicle are more frequent and sometimes more brutal than those observed in airplane (i.e. in particular in bends, when changing lanes or braking). Driving a vehicle takes place in traffic that can sometimes create very complex situations. The driver is thus more solicited for the control of his vehicle than is an airplane pilot. The sensory informations used for driving a vehicle are greater and sometimes different that the ones used for flying an airplane.

This algorithms are based on two main principles:

- The first so called "Washout", the platform is linearly moved in the same acceleration direction to reproduce the transient longitudinal and lateral accelerations and return back to its neutral position. This last movement should not be detected by the driver (with respect to the sensation threshold) to be not interpreted as a false cue [9],

- The second so called "tilt-coordination", allows the restitution of sustained longitudinal and lateral accelerations by tilting the cabin forward or backward to gain a component of gravity vector. Such tilt can be interpreted by his/her vestibular system as either a positive or negative acceleration, depending on the direction of the tilt. The rate of tilting must be done under the detectable threshold of semicircular channels [9].

The design of cueing algorithms in the field of the driving simulation is a complicated task, it depends on simulator architecture and the type of maneuver we are looking to reproduce [2], [3], [4], [7]. However, the classical approach suffers from a certain number of disadvantages (not taken into account of human perception, the wish of reproduce the movement and not the sensation of driving, etc). For this, several improvements were proposed mainly for the adaptive and optimal approaches. The first technique (adaptive approach) [10], [11], [12] combines nonlinear filters used jointly with a control method optimizing the error between the state of the simulated vehicle and the state of the platform, which makes it possible to adjust the profits and the parameters of the filters to reduce the space of movement. The second technique (optimal approach) fills the defects of the first method and takes into account the human perception aspect by minimizing the error of the feeling perceived 
between the dynamics ones of the simulated vehicle and of the platform. In fact, a great space of movements is requires [13], [14], [15].

For the current study, a low-cost motion platform equipped with two degrees of freedom have been designed and built. The choice of this architecture is based on the simplicity of design, type of phsycophysic studies to be carried out and the global cost. It is possible to move the simulator's cab with a longitudinal movement, with a small rotation movement of the driver seat.

\section{Platform Modelling}

To model the driving simulator motion, the overall system is considered as two independent systems mechanically linked: the rotating driving seat and the longitudinal motion platform (cabin). Each of them is driven by a single actuator and a screw/nut device. The motion platform undergoes translational motions according to one direction (front and back) which correspond to driver's acceleration and deceleration. The overall system's design allows having a simple linear model of the motion.

The choice of the types of motors and screw/nut tread device is taken according to many parameters, that is, accelerations to reproduce, delivered torque, nominal rotational rates and thermic dissipation.

\section{A. The linear motion platform}

The motion base supports the cabin which consists of the seat, the vehicle chassis and the driver. Because the rotations of the seat are slow and low amplitude, its induced inertia is negligible comparing to the total mass of the cabin's set. The linear motion of the cabin's set is made thanks to a ball screw/nut transmission mechanism driven by a DC actuator. The technological design was made in order to reduce, mechanical flaws, static and dynamic friction, and to facilitate the design of simple controllers. The overall modelisation was detailed in previous papers [16], [17], we remember here only the dynamic model of the cabin's position $X(s)$ and the voltage command signal $U(s)$ :

$$
\frac{X}{U}=\frac{1}{s} \frac{K_{t 1}}{\left(J_{1} s+f_{1}\right)\left(L_{1} s+R_{1}\right)+\frac{2 \pi N_{1}}{p_{1}} K_{e 1} K_{t 1}}
$$

where: $s$ is the Laplacien operator. $K_{t 1}, K_{e 1}$ are electrical constant of platform DC motor. $J_{1}, f_{1}$ global rotational inertia and friction of platform. $R_{1}, L_{1}$ are platform motor armature resistance and inductance. $N_{1}, p_{1}$ are reduction factor and screw thread of screw/nut device.

\section{B. The rotating seat model}

The driver seat can perform two kinds of small rotation movements; the rotation of only the seat's back or the entire seat rotation. This is achieved by a single actuator with a manual switch, which is configured to perform either of two movements, but not both at time. This motion can be coupled to the linear one giving five possible combinations for experimental investigations of cue strategy as:
- Linear movement of platform + rotation of the entire seat

- Linear movement of platform + rotation of only seat's back

- Only the linear movement

- Only rotation of entire seat

- Only rotation of seat's back

Using a modelling approach similar to that of cabin supporting platform described in [17], we obtain the dynamic equation model of the seat, as follows:

$$
\ddot{\theta}=\frac{m_{b} g \rho_{1} \sin (\phi+\theta)-\ddot{x} \rho_{1} \cos (\phi+\theta)}{\left(J_{b}+m_{b} \rho_{1}^{2}\right)}
$$

where, $m_{b}, J_{b}$ : driver's bust mass and inertia, $\rho_{1}$ : distance between the bust centre of gravity and the bust rotation axis, $\ddot{x}, \ddot{\theta}$ : platform longitudinal acceleration and the driver's bust angular acceleration, $\theta$ : angular position of driver's bust, $\phi$ : initial angular position of driver's bust centre of gravity. From equation (2), we distinguish between the platform longitudinal acceleration effect and the gravity effect. Consequently, our interest is the relation between the angular and longitudinal accelerations (figure 1). So, from the above equation, we have:

$$
\left(J_{b}+m_{b} \rho_{1}^{2}\right) \ddot{\theta}=-\ddot{x} \rho_{1} \cos (\phi+\theta)
$$

This equation shows the inertial moment which is induced from the platform acceleration. This moment causes an angular acceleration which animate the driver's bust in the opposite direction of the platform acceleration. By the aid of an algorithm of motion cue, we can restitute the calculated angular acceleration, by tilting the seat's back, with a scale factor $k_{s}$, as:

$$
\ddot{\theta}=k_{s} \frac{\ddot{x} m_{b} \rho_{1} \cos (\phi+\theta)}{J_{b}+m_{b} \rho_{1}^{2}}
$$

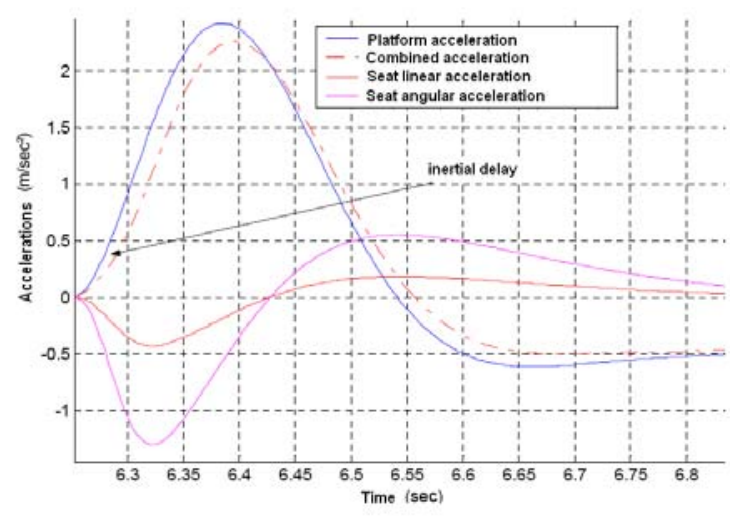

Fig. 1. Seat's inertial cue

\section{WAShOUT FILTER}

As we state previously, the platform has 2 DOF the longitudinal movement and the seat rotations. These rotations 
are made to improve the movement perception, but not in any case could be used for a tilting strategy in coordination with longitudinal movements.

\section{A. Classical Algorithm}

This algorithm use a linear high-pass filters to reproduce the transient accelerations of platform. The acceleration of the simulated vehicle is passed though this filters to remove the sustained components which take the platform over its physical limits. The resulting signal is integrated twice and passed through a second high pass filter to produce the position reference for the actuators as shown in figure 2 . The choice of this filters (order and parameters) depend on

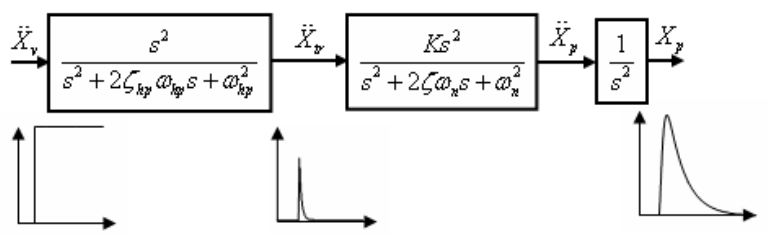

Fig. 2. Classical Washout Algorithm for longitudinal motion

the architecture of the driving simulator and the types of maneuvers executed by the driver. It must respect some of perception and technological constraints, such, the absolute maximum displacement allowed by the architecture of the platform, the relative maximum displacement of the platform in response to a simulated acceleration over a time $t_{\max }$, the acceleration and velocity thresholds that must be not exceeded during the Washout and the technological limitations of the platform's actuator (response time and friction). Consequently, we can suppose that the output of the washout filter is the impulse response of a second low-pass filter as:

$$
\frac{X_{p}(s)}{\ddot{X}_{t r}(s)}=\frac{K}{s^{2}+2 \zeta \omega_{n} s+\omega_{n}^{2}}
$$

where $X_{p}(s)$ : Platform position, $\ddot{X}_{t r}(s)$ : The transitory acceleration filtered by the first high pass-filter, $\zeta:$ The damping coefficient, $\omega_{n}$ : The natural pulsation, $K$ : scaling factor. The impulse response of this filter is given by:

$$
h(t)=\frac{K}{\tau_{1}-\tau_{2}}\left[\exp \left(-\frac{t}{\tau_{1}}\right)-\exp \left(-\frac{t}{\tau_{2}}\right)\right]
$$

where

$\tau_{1,2}=\frac{\zeta}{\omega_{n}} \pm \frac{\sqrt{\zeta^{2}-1}}{\omega_{n}}$ with $\zeta>1$ From this equation, we can deduce the maximum achieved position, the maximum velocity and acceleration of retuning back to the neutral position for a given transient acceleration, as:

$$
\begin{gathered}
X_{p \max }=K \omega_{n} \xi<P_{\max } \\
\dot{X}_{p \max }=K \omega_{n}^{2} \xi^{2}<v_{s} \\
\ddot{X}_{p \max }=K \omega_{n}^{3} \xi^{3}<a_{s}
\end{gathered}
$$

with

$$
\xi=\exp \left[\frac{\zeta}{\sqrt{\zeta^{2}-1}} \ln \left(\zeta-\sqrt{\zeta^{2}-1}\right)\right]
$$

where, $P_{\max }, v_{s}, a_{s}$ : are respectively, the maximum displacement allowed by the platform, the velocity and acceleration perception thresholds (figure 3). Finally, the last constraint is purely technological and concerns the proper friction of simulation, and consequently, its direct dependency on the parameters of actuators and Washout filter(this two last blocs are in cascade). Precisely, in order to profit at maximum of actuator characteristics (synthesized during initial dimensioning), the Washout filter must be selected in a manner to minimize the total friction. To be done, the following condition must be respected:

$$
2 \zeta \omega_{n}<f_{0}
$$

where $f_{0}$ is the proper firction of the actuator system. If this condition is not satisfied (if the actuator's proper friction less than the filter one), the simulation system is depending only on the actuator parameter, and consequently, the tuning of motion cueing algorithm is reduced even eliminated.

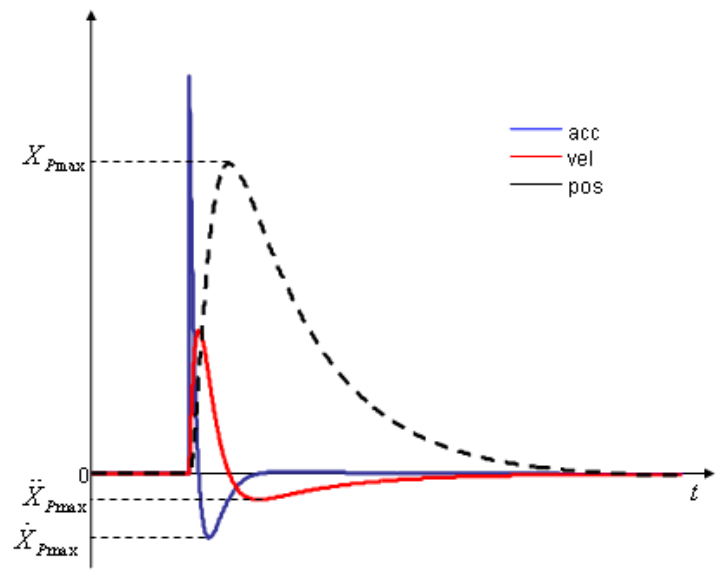

Fig. 3. The maximum response of the longitudinal Washout filter

Figure 4 shows the selected parameters of the natural frequency $\omega_{n}$ with respect of a set of damping parameters $\zeta$, with taking all the constraints montionned above (in position, velocity and acceleration thresholds, and proper actuator's friction).

\section{B. Optimal and Adaptive Algorithm}

These two motion cueing strategies are both the most popular techniques control for driving simulator. For more informations we invite the reader to consult our work in [18].

\section{IMPLEMENTATION}

In order to compare the performances of the longitudinal classical algorithm with the adaptive and optimal approaches, experimentations are carried out on the present driving simulator. Virtual scenes are projected by a three Barco projectors on a fixed wide screen. Traffic simulation, sound rendering and scenarios administration are computed by ARCHISIM 


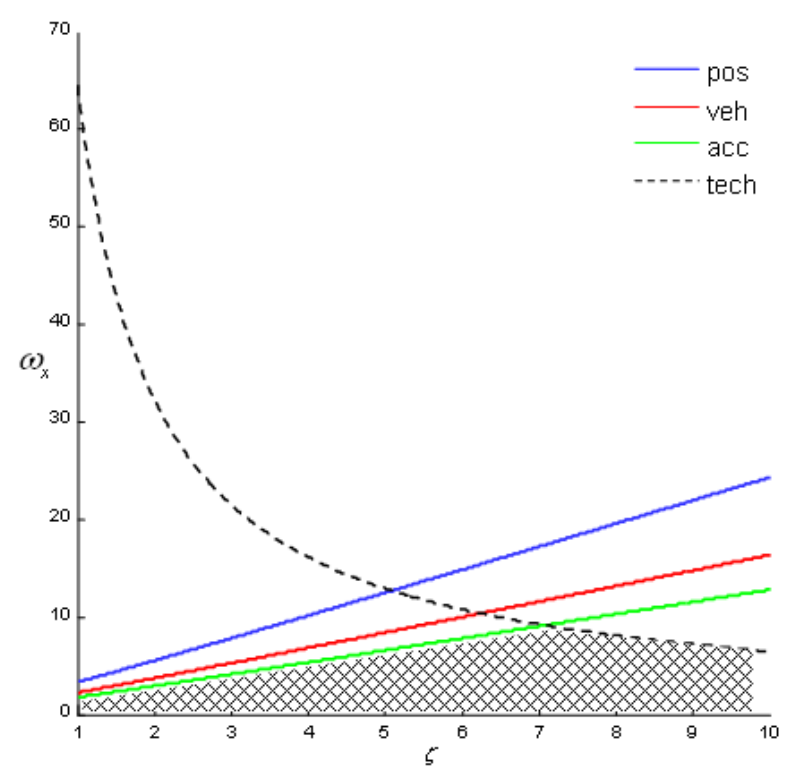

Fig. 4. The Washout filter parameters within respect to the constraints

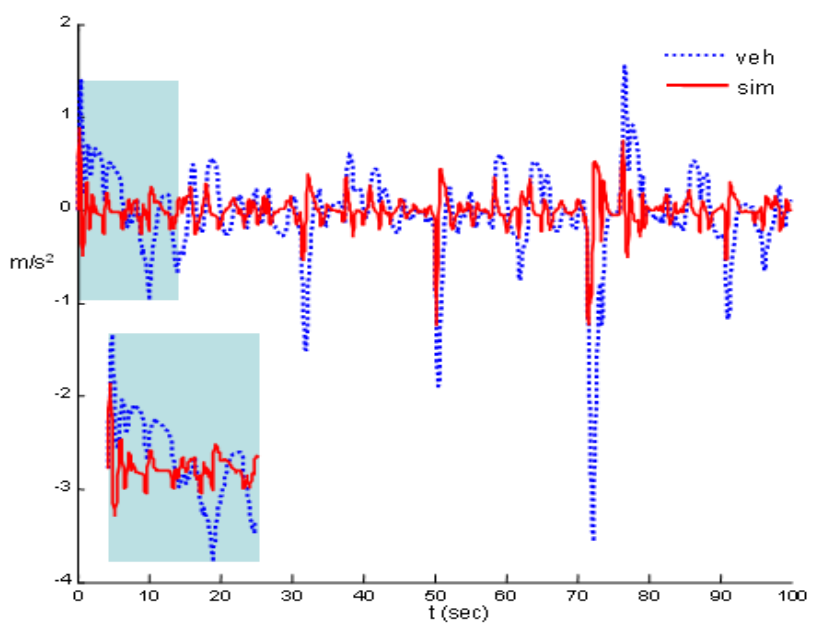

Fig. 5. Cabin's accelerations using classical algorithm

Software [19]. First, a scenario consisting in a set of accelerations, decelerations and braking is accomplished. The resulting acceleration from the virtual vehicle dynamic model is saved to be used later for the classic, adaptive and optimal algorithms. This is done to compare the different algorithms for the same maneuver. The parameters of each algorithm are adjusted to respect the physical constraints of the platform $\left( \pm 0.6[\mathrm{~m}], \pm 1.3 \mathrm{~g}\left[\mathrm{~m} / \mathrm{s}^{2}\right]\right.$ ). For the optimal method, we use an otolith model of second order proposed by Young and al. in [20]. Figure 5 shows the vehicle and simulator acceleration, where figure 6 illustrates the Cabin's position using two sets of classical Washout filter parameters. Figures (7, 8) show the vehicle and the simulator accelerations using the longitudinal adaptive and optimal approaches respectively. In figure 9 we give the cabin's position using the longitudinal adaptive and optimal approaches. So, the classical algorithm gives a good results except for the brutal braking or onset

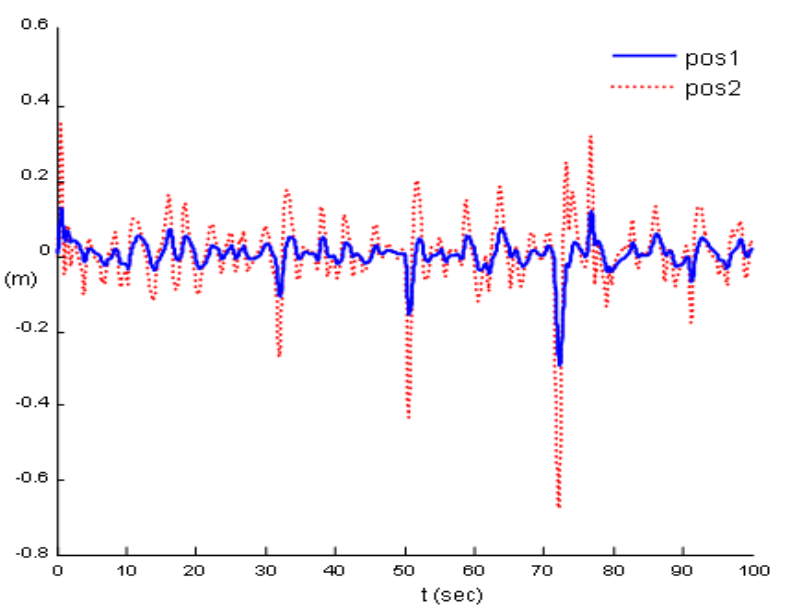

Fig. 6. Cabin's position using classical algorithm

acceleration, where it presents a detectable false cue. This is because the filter parameters are ajusted for an absolute maximum displacement $(K=1)$, where the onset acceleration and braking present a high values, which require a tilting strategy to be restituted. In fact, for the same restituted acceleration, the Washout filter with the first set of parameters (pos1 in figure 6) require less displacement then the one of the second set of parameters (pos2 in figure 6), this because the second set don't respect the position constraint. In addition, we had implemented the adaptive and the optimal approaches for the longitudinal movement. We found that the optimal algorithm provide better onset cueing than the adaptive one,

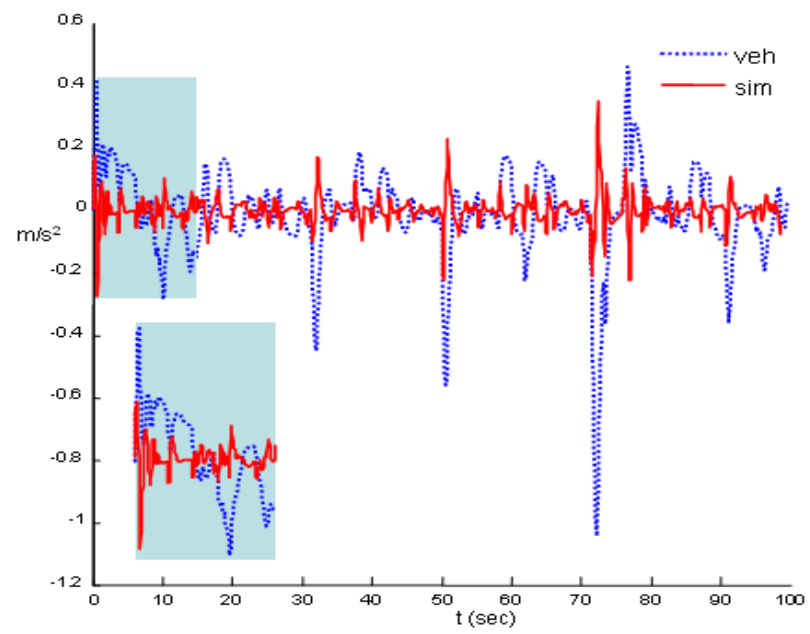

Fig. 7. Cabin's accelerations using adaptive algorithm

but the adaptive algorithm reduce more the false cues than the optimal one. An interessent remark, that for the same order of value of restituted acceleration, the optimal algorithm require more displacement than the adaptive one, more adjustement of the penality coefficients of the adaptive cost function would improve the acceleration cueing. Finally, the tuning of the classical approach is more easier and objective than the 


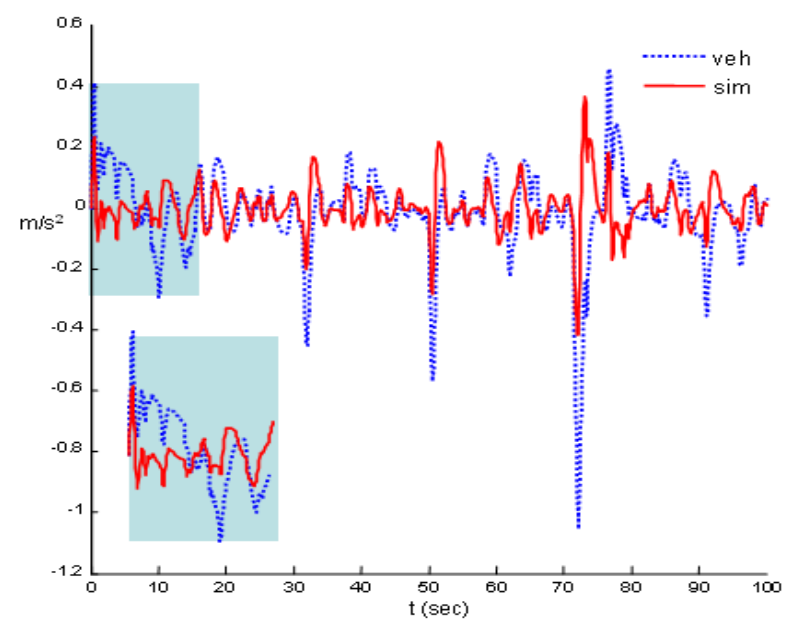

Fig. 8. Cabin's accelerations using optimal algorithms

two others. The optimal algorithm require a great workspace and the adaptive one is more difficult to tune. For these reasons (simplicity of tuning and implementation), we retain the classic Washout filter for the experimetation.

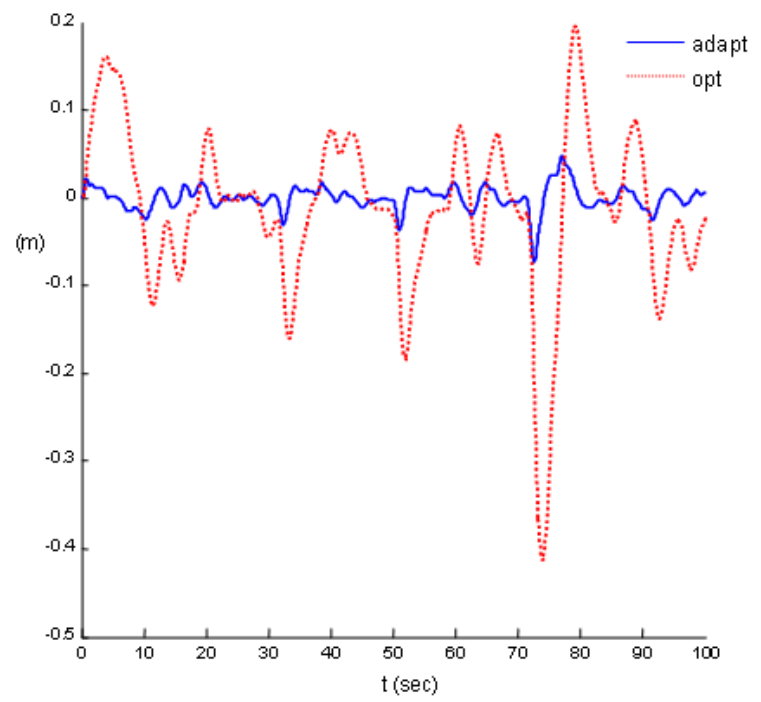

Fig. 9. Cabin's positions using adaptive and optimal algorithms

\section{PSYCHOPHYSICAL EXPERIMENT}

In the psychophysical experiment, we will try to explore the various movements accessible by the platform in order to define the configuration of movement which makes it possible to produce a minimal inertial effect sufficient enough to improve driving on the simulator. With this intention, we are interested in acceleration and braking control in order to regulate simulated vehicle speed during the follow-up of a "lead car". Generally, such a situation constrains the driver to readjust his/her speed according to the speed variations of the "lead car". In order for speed adjustment to be possible, the driver relies on the information perceived by the vestibular system on the one hand, and on the information perceived by the visual channel on the other.

\section{A. Experimental Conditions}

6 movement conditions have been proposed for platform movement:

- Without movement (W-Off) : no movement is activated on the platform

- Long platform movement (L-Off) : only longitudinal movement is activated. The displacement and the maximum acceleration of the platform are $30 \mathrm{~cm}$ et $0.4 \mathrm{~g}$ respectively.

- Short platform movement (S-Off) : Only longitudinal movement is activated. The displacement and maximum acceleration of the platform are $10 \mathrm{~cm}$ et $0.2 \mathrm{~g}$ respectively.

- Seat movement (W-On) : Only longitudinal movement is activated. The displacement and maximum acceleration of the platform we have noted are $4 \mathrm{rad} / \mathrm{s}^{2}$ et $6 \mathrm{deg}$ respectively.

- Long platform movement combined with seat movement (L-On) : Platform and seat movement are activated.

- Long platform movement combined with seat movement (S-On) : Platform and seat movement are activated.

\section{B. Driving Simulator}

32 people participated to the experiment, they drove in a moving-base driving simulator (INRETS MSIS SIM class), with dynamic and interactive visual image. The drivers habits related to the driving activity were investigated by Manchester driving behaviour questionnaire (MDBQ). The main subjective defendant variables recorded was the rank allocated to each conditions. We also considered the comments of the drivers as regards the realism of deceleration, acceleration and braking maneuver. The objective dependent variables recorded were the mean headway time (HT) and the variation of decelerations (VARdec). HT refers to the delay between the lead vehicle and the simulator. VARdec refers to the changes of deceleration of the piloted vehicle.

\section{Results}

The main objective of this research was to assess the relevance of our driving simulator architecture choice (longitudinal + back of seat motion) and to compare different modalities for longitudinal accelerations rendering. A secondary aim was to support the use of individual characteristic measures as potential indices for the assessment of new driving simulators. It appears that the longitudinal displacement of the motion-base alone, is not sufficient to modulate the driving performances in comparison to the lack of platform motion. However the tilt of the seat back coupled to longitudinal movement provides information that modulate them. The HT in S-On had decreased significantly regarding the other situations conditions. we can interpret 
this result as an increase of confidence and may be as an increase of the virtual vehicle control. We also remind that this condition is subjectively considered as the better among the six experimental conditions proposed in our experiment. Such interpretation is reinforced by the fact that the MDBQ individual parameter offers a same kind of result, but for prudent drivers exclusively.

\section{CONCLUSION}

In this paper, three algorithms (Classical, Adaptive and Optimal) for motion cueing are exposed and experimented on our low cost platform. The implementation concerns only the longitudinal acceleration restitution. The aim of this study is to compare the performances of each algorithm, and its impact on the driver perception.

We presented an approach for the selecting of Classical Washout filters which may respect the platform constraints and perception thresholds. The classical Washout is the more appropriate for our case in term of human perception and design simplicity. The parameters adjustment is too easy regarding the remaining algorithms. The inconvenience of this method is that some false cues are induced for the brutal changes in acceleration like braking, due to the linear characteristic of the high-pass filter, and that the gain of filter is selected for the worst case.

Secondly, adaptive algorithm allows the adjustment of the filter parameters at each time of the driving simulation. This can reduce some false cues generated by the classical algorithm. Nevertheless, we have found that returning back to the neutral position is more slow then the classical algorithm, the perception is more soft mainly at the onset of acceleration. The inconvenience of this method is the difficulty to find the most relevant weighting of the cost function, and initial values of the different parameters, that gives the best results while assuring stability.

Optimal algorithm present different lacks. Despite, it minimizes the sensation error between the driver on the virtual vehicle, and the one on the simulator platform. The obtained results are not sufficient to cue a good perception. This due to the physical constraints of our simulator.

Based on the psychophysical experiments, we have shown that it was possible to propose a low-cost driving simulator for studies dedicated to platooning situations. According to the task and the environmental specificities, the efficiency of the amplitude of reproduced longitudinal motion seems to vary. More precisely, from a subjective point of view, short longitudinal motion of the platform associated with a tilt of the back of the seat seems to be appropriate for normal traffic situations, while long motions seem more convincing for the emergency braking. Long motion might be then, specifically employed to study driver assistance systems like the E.B.A. (Emergency Braking Assistance) for example. However to explore the prototype capacity driving improvement more that we carried out, other algorithms and other movement configuration should be tested. Consequently, other experiments psychophysical should be carry out because it is the unique objective manner to evaluate the simulator fidelity.

\section{ACKNOWLEDGMENT}

This work was supported partially by the French National Agency of Research (ANR), within the framework of PREDIT project. These last are focused on the road safety.

\section{REFERENCES}

[1] A. Kemeny, "Simulation et perception du mouvement," in Driving Simulation Conference(DSC99), Paris, France, July 1999, pp. 33-55.

[2] S. F. Schmidt and B. Conrad, "Motion drive signals for piloted flight simulators," in Contractor Report NASA CR-1601, Washington, USA, 1970.

[3] W. R. Sturgeon, "Controllers for aircraft motion simulators," Journal of Guidance and Dynamics, vol. 4, pp. 184-191, Mar.-Apr. 1981.

[4] P. R. Grant and L. D. Reid, "Motion washout filter tuning: Rules and requirements," Journal Of Aircraft, vol. 34, pp. 145-151, Mar.-Apr. 1997.

[5] M. Idan and M. A. Nahon, "Off-line comparison of classical and robust flight simulator motion control," Journal of Guidance and Dynamics, vol. 22, pp. 702-709, Sept.-Oct. 1999.

[6] L. D. Reid and M. A. Nahon, "Response of airline pilots to variations in flight simulator motion algorithms," Journal Of Aircraft, vol. 7, pp. 639-646, July 1988.

[7] M. A. Nahon and L. D. Reid, "Simulator motion-drive algorithms: A designer's perspective," Journal of Guidance and Dynamics, vol. 13, pp. 356-362, July 1989.

[8] G. Reymond, "Contribution respective des stimuli visuels, vestibulaires et proprioceptifs dans la perception du mouvement du conducteur," in Paris6 PhD Thesis), Paris, France, Dec. 2000.

[9] G. Reymond and A.Kemeny, "Motion cueing in the renault driving simulator," in Vehicule System Dynamic, vol. 34, Paris, France, Oct. 2000, pp. 249-259.

[10] R. V. Parrish, J. E. Dieudonne, R. L. Bowles, and D. J. Martin, "Coordinated adaptive washout for motion simulators," Journal of Aircraft, vol. 12, pp. 44-50, July 1975.

[11] M. A. Nahon, L. D. Reid, and J. Kirdeikist, "Adaptive simulator motion software with supervisory control," Journal of Guidance and Dynamics, vol. 15, pp. 376-383, Mar.-Apr. 1992.

[12] D. Ariel and R. Sivan, "False cue reduction in moving flight simulators," IEEE Transactions on Systems, Man and Cybernetic, vol. 14, pp. 665-671, July-Aug. 1984.

[13] R. Sivan, J. Ish-shalom, and J. . Huang., "An optimal control approach to the design of moving flight simulators," IEEE Transactions on Systems, Man and Cybernetic, vol. 12, pp. 818-827, July-Aug. 1982.

[14] F. Cardullo, R. Telban, and J. Houck, "Motion cueing algorithms: A human centered appraoch," in 5th International Symposium on Aeronautical Sciences, Zhukovsky, Russia, 1999.

[15] R. Telban and F. Cardullo, "A nonlinear human centred approach to motion cueing with neurocomputing solver," in AIAA Modeling and Simulation Technologies Conference, 2002.

[16] H. Mohellebi, S. Espie, H. Arioui, A. Amouri, and A. Kheddar, "Low cost motion platform for driving simulator," in 5th International Conference on Machine Automation (ICMA04), Osaka, Japon, Nov. 2004.

[17] H. Arioui, L. Nehaoua, and A. Amouri, "Classic and adaptive washout comparaison for a low cost driving simulator," in Proceedings of the 13th Mediterranean Conference on Control and Automation (MED05), Limassol, Cyprus, June 2005, pp. 586-591.

[18] L. Nehaoua, H. Arioui, H. Mouhellebi, and S. Espie, "Motion cueing algorithms for small driving simulator," in 2006 IEEE International Conference In Robotics and Automation (ICRA06), Orlando, Florida, May 2006.

[19] S. Espie, "Vehicle-driven simulator versus traffic-driven simulator: the inrets approach-driving simulation conference," in Driving Simulation Conference (DSC99), Paris, France, July 1999.

[20] L. Young and J. L. Meiry, "A revised dynamic otolith model," in Aerospace Medicine, vol. 39, June 1968, pp. 606-608. 